\title{
Tabagismo domiciliar em famílias com crianças menores de 5 anos no Brasil
}

\author{
Regina M. V. Gonçalves-Silva, ${ }^{1}$ Joaquim G. Valente, ${ }^{2}$ \\ Márcia G. F. Lemos-Santos ${ }^{1}$ e Rosely Sichieri²
}

Como citar Gonçalves-Silva RMV, Valente JG, Lemos-Santos MGF, Sichieri R. Tabagismo domiciliar em famílias com crianças menores de 5 anos no Brasil. Rev Panam Salud Publica. 2005;17(3):163-9.

RESUMO Objetivo. Estimar a prevalência e identificar os principais determinantes da exposição ao tabagismo domiciliar em familias com crianças menores de 5 anos.

Métodos. Estudo transversal, de base populacional, com 2037 crianças e seus respectivos responsáveis na Cidade de Cuiabá, Estado de Mato Grosso, Brasil. Os pais ou responsáveis responderam um questionário contendo perguntas relacionadas ao hábito de fumar dos moradores, às características sociodemográficas da família e às suas condições de moradia.

Resultados. A prevalência de tabagismo domiciliar foi de 37,7\%. Para os domicilios com renda per capita mensal de 2 ou mais salários mínimos, a prevalência de tabagismo foi de $24 \%, 1$, contra 31,3\% nos domicilios com renda per capita mensal entre 1 e 1,9 salário mínimo e 46,0\% nos domicilios com renda inferior a 1 salário mínimo. Quanto mais baixo o nível socioeconômico das familias, maior a prevalência de tabagismo domiciliar $(26,9 \%, 26,4 \%$, $34,9 \%, 45,1 \%$ e 47,2\% para as classes $A, B, C, D$ e E, respectivamente). Maior prevalência também foi observada quando o pai não morava no domicílio (47,5 versus $35,5 \%$ ), quando o chefe da familia era uma pessoa diferente do pai ou da mãe (53,8 versus 33,9 e 38,8\%) e quando o pai e a mãe tinham menos de 20 anos $(46,1$ versus $22,5 \%$ aos 40 anos para as mães e 52,5 versus $31,2 \%$ aos 40 anos para os pais). Em relação à ocupação, a menor prevalência de tabagismo no domicilio foi observada em profissionais de saúde e professores, tanto para mulheres $(18,3 \%)$ quanto para homens $(14,7 \%)$. Permaneceram associadas com o tabagismo no domicílio, após a regressão logística multivariada, a presença do pai no domicílio, a escolaridade e a ocupação do pai e da mãe, a idade da criança e a renda per capita mensal da família. Conclusão. O risco de exposição ao tabagismo domiciliar e, portanto, ao tabagismo passivo nesta amostra de crianças menores de 5 anos foi maior em familias de menor nível socioeconômico.

Palavras chave Tabagismo, emprego, escolaridade, fatores socioeconômicos.

1 Universidade Federal de Mato Grosso (UFMT), Faculdade de Nutrição, Departamento de Alimentos e Nutrição. Enviar correspondência para Regina M. V. Gonçalves-Silva no seguinte endereço: Rua 27, casa 207, CEP 78068-590, Cuiabá, MT, Brasil. Telefone: +55-65-664-3104; fax: +55-65-3052-2202; e-mail: reveras@uol.com.br

2 Universidade do Estado do Rio de Janeiro (UERJ), Instituto de Medicina Social.
A fumaça lançada no ambiente pelo cigarro dos fumantes é o mais importante poluidor do ar domiciliar. Ela contém todos os constituintes nocivos do cigarro, sendo que o fumante passivo sofre danos à saúde semelhantes aos que sofre o fumante (1).
Dados os efeitos nocivos do cigarro, existe um interesse internacional crescente na concentração de esforços para reduzir a prevalência do tabagismo (2). A literatura mostra que os fatores sociodemográficos, especialmente a idade, o sexo, a escolaridade e o nível 
socioeconômico, são importantes na determinação da iniciação e manutenção do hábito de fumar na população em geral $(3,4)$. Observa-se também uma associação negativa entre o tabagismo e a qualificação das ocupações profissionais em termos de nível de especialização (5).

Os menores de 5 anos de idade são mais vulneráveis do que as crianças de outros grupos etários aos efeitos deletérios do fumo, principalmente às enfermidades respiratórias (6). Por serem menores e respirarem mais rapidamente, inalam maior quantidade de substâncias químicas danosas aos pulmões por quilograma de peso corporal. Além disso, o aparelho respiratório das crianças nessa faixa etária está menos desenvolvido, o que as torna mais suscetíveis às infecções (7). Contudo, é pouco explorado o tema dos fatores determinantes da exposição ao fumo em domicílios onde vivem crianças menores de 5 anos. $\mathrm{O}$ objetivo do presente estudo foi estimar a prevalência e identificar os principais determinantes da exposição ao tabagismo domiciliar em famílias com crianças menores de 5 anos na Cidade de Cuiabá, Brasil.

\section{MATERIAIS E MÉTODOS}

Foi realizado um estudo transversal de base populacional. A amostra foi constituída pelas crianças vacinadas de agosto de 1999 a janeiro de 2000 nos postos de saúde de Cuiabá, capital do Estado de Mato Grosso, Brasil. O Município de Cuiabá, com um território de 3 224,68 $\mathrm{km}^{2}$, está localizado na Região Centro-Oeste. Na época do estudo, a população contabilizava 483 346 habitantes, dentre os quais 43197 eram menores de 5 anos (8). Conforme as informações da Secretaria Municipal da Saúde, a cobertura vacinal em Cuiabá é alta (97\% para tríplice bacteriana em menores de 1 ano). As crianças são encaminhadas pelo hospital em que nasceram ao posto de saúde mais próximo de sua residência para imunização.

No período em que foi realizada a pesquisa, Cuiabá contava com 38 pos- tos de saúde, dos quais foram sorteados 10, proporcionalmente ao número de doses de vacina aplicadas. Em cada um desses 10 postos, foram sorteadas aproximadamente 200 crianças (dependendo do fluxo de crianças no dia), cujos responsáveis foram convidados a participar do estudo. Dos 2045 responsáveis convidados, oito $(0,4 \%)$ negaram-se a responder o questionário, tendo sido incluídas na pesquisa 2037 famílias.

O tabagismo domiciliar foi definido como a presença de pelo menos um morador fumante no domicílio.

A classificação da Associação Brasileira de Empresas de Pesquisa (ABEP) foi utilizada para determinar o nível socioeconômico das famílias. A classificação da ABEP se baseia na acumulação de bens materiais, no poder aquisitivo e na escolaridade do chefe da família (9). Essa classificação enquadra as famílias em cinco níveis socioeconômicos, de A (mais elevado) a E (mais baixo). A renda mensal per capita da família, expressa em salários mínimos, foi obtida através da soma das rendas individuais dos moradores dividida pelo total de moradores do domicílio. Na época do estudo, o valor do salário mínimo era de 136 reais (72 dólares).

A escolaridade dos pais foi classificada em quatro categorias: nível superior completo ou mais; nível médio completo; nível fundamental completo; e nível fundamental incompleto ou analfabeto. Para a classificação da atividade profissional, tomou-se como parâmetro a Classificação Brasileira de Ocupações (10).

A ocupação dos pais foi classificada em ocupações técnicas e artísticas (profissionais de saúde e professores e outros profissionais); diretores e gerentes de empresa; administração; comércio; serventia, hospedagem, higiene e segurança; agropecuária; produção industrial e transportes (motoristas e ajudantes, trabalhadores da construção civil, mecânicos e ajudantes, operadores de máquinas e demais profissionais); e ocupações não-remuneradas (estudantes, desempregados e aposentados). Em relação às mães, a ocupação foi classificada em ocupações técnicas e artísticas (profissionais de saúde e professoras e outras profissionais); administração; comércio, serventia, hospedagem, higiene e segurança (empregadas domésticas e demais profissionais); produção industrial; e ocupações não-remuneradas. Nesta última categoria, além das estudantes, desempregadas e aposentadas, foram incluídas, como um grupo separado, as donas-de-casa, já que esta foi a ocupação declarada por um grande número de mães, sendo elevada a prevalência de tabagismo nessas mulheres. Para fins de análise, foram agregados os grupos ocupacionais com prevalência semelhante de tabagismo (ou seja, diferença de menos de 5\%).

Para analisar de forma combinada a ocupação e a escolaridade, foi criada uma variável binária para a escolaridade (mais baixa e mais alta), com pontos de corte específicos para cada categoria ocupacional. Para as mães, a escolaridade mais baixa e a mais alta, respectivamente, consideradas para as ocupações de saúde e ensino (categoria de referência) foram: ensino médio completo e ensino superior completo; domésticas: até $7^{\mathrm{a}}$ série do ensino fundamental e ensino fundamental a médio completo; donas-de-casa: até $7^{\mathrm{a}}$ série do ensino fundamental e ensino fundamental a superior completo; e demais profissionais: até ensino fundamental completo e ensino médio a superior completo. Para os pais, a escolaridade mais baixa e a mais alta, respectivamente, consideradas para as ocupações técnicas e artísticas (categoria de referência) foram: até ensino fundamental completo e ensino médio a superior completo; ocupações na área de administração, comércio, serventia e segurança: até ensino fundamental completo e ensino médio completo ou mais; e indústria: até $7^{\mathrm{a}}$ série do ensino fundamental e ensino fundamental a médio completo.

A razão de prevalências (RP) e o intervalo de confiança de 95\% (IC95\%) foram as medidas de associação utilizadas. A análise multivariada foi realizada por regressão logística. Para a exclusão das variáveis, além da significância estatística $(P<0,05)$, observouse o efeito que sua remoção produzia 
nas estimativas pontuais e no IC das variáveis remanescentes no modelo. Os dados foram analisados com os programas Epi-Info, versão 6.02, e Statistical Package for the Social Sciences (SPSS), versão 9.0 .

O estudo foi aprovado pelo comitê de ética da Universidade Federal de Mato Grosso (UFMT). O consentimento informado foi obtido dos pais das crianças que participaram da pesquisa.

\section{RESULTADOS}

Dentre as 2037 crianças participantes, $51,4 \%$ eram do sexo masculino e $48,6 \%$ do sexo feminino. A prevalência global do tabagismo domiciliar foi de $37,7 \%$.

A prevalência de tabagismo no domicílio foi maior nas casas onde as crianças eram mais velhas (36 meses ou mais), quando o pai não morava com a criança e quando o chefe da casa era uma pessoa que não o pai ou a mãe da criança. Piores condições de moradia (material da casa, tipo de abastecimento de água e da água utilizada para beber, tipo de esgotamento sanitário, coleta de lixo e número de banheiros na casa) associaram-se à maior prevalência de tabagismo no domicílio (tabela 1). Detectou-se tendência linear significativa e direta entre a prevalência do hábito de fumar e a idade da criança, e tendência linear significativa e inversa entre a prevalência de tabagismo e a escolaridade da mãe e do pai da criança, a renda mensal per capita da família e o nível socioeconômico.

A ocupação dos pais e as respectivas taxas de prevalência de tabagismo domiciliar e RP estão apresentadas na tabela 2. Dentre as ocupações referidas pelas mães, o grupo das empregadas domésticas foi o que apresentou a maior prevalência de tabagismo. A prevalência de tabagismo também foi elevada entre as mães que se declararam sem ocupação remunerada e como sendo donas-de-casa. Em relação à ocupação do pai, observou-se que apenas os professores e os profissionais da saúde apresentaram prevalência de tabagismo mais baixa do que os demais grupos ocupacionais.
TABELA 1. Tabagismo no domicílio ${ }^{a}$ segundo a idade da criança, a condição sociodemográfica da família e as condições ambientais, Cuiabá (MT), Brasil, 1999

\begin{tabular}{|c|c|c|c|}
\hline Variável & Fumantes/n & Prevalência (\%) & $\mathrm{RP}(\mathrm{IC} 95 \%)^{\mathrm{b}}$ \\
\hline \multicolumn{4}{|l|}{ Idade da criança ${ }^{c}$} \\
\hline$<12$ meses & $342 / 1006$ & 34,0 & 1,00 \\
\hline 12 a 35 meses & 268/663 & 40,4 & $1,19(1,05$ a 1,35$)$ \\
\hline 36 meses ou mais & $158 / 368$ & 42,9 & $1,26(1,09$ a 1,46$)$ \\
\hline \multicolumn{4}{|l|}{ Pai mora com a criança } \\
\hline Sim & $588 / 1658$ & 35,5 & 1,00 \\
\hline Não & $180 / 379$ & 47,5 & $1,34(1,18$ a 1,52$)$ \\
\hline \multicolumn{4}{|l|}{ Chefe da casa } \\
\hline Pai & $517 / 1523$ & 33,9 & 1,00 \\
\hline Mãe & $66 / 170$ & 38,8 & $1,14(0,94$ a 1,40$)$ \\
\hline Outros & $185 / 344$ & 53,8 & $1,59(1,40$ a 1,79$)$ \\
\hline \multicolumn{4}{|l|}{ Idade da mãe da criança ${ }^{d}$} \\
\hline$<20$ & $153 / 332$ & 46,1 & 1,00 \\
\hline 20 a 29 & $453 / 1224$ & 37,0 & $0,80(0,70$ a 0,92$)$ \\
\hline 30 a 39 & $146 / 421$ & 34,7 & $0,75(0,63$ a 0,90$)$ \\
\hline 40 e mais & $9 / 40$ & 22,5 & $0,49(0,22$ a 0,73$)$ \\
\hline \multicolumn{4}{|l|}{ Idade do pai da criança ${ }^{d}$} \\
\hline$<20$ & $23 / 44$ & 52,3 & 1,00 \\
\hline 20 a 29 & $299 / 859$ & 34,8 & $0,67(0,49$ a 0,90$)$ \\
\hline 30 a 39 & $218 / 601$ & 36,3 & $0,69(0,51$ a 0,94$)$ \\
\hline 40 e mais & $48 / 154$ & 31,2 & $0,60(0,41$ a 0,86$)$ \\
\hline \multicolumn{4}{|l|}{ Escolaridade da mãe ${ }^{c}$} \\
\hline Superior completo ou mais & $24 / 129$ & 18,6 & 1,00 \\
\hline Médio completo & $162 / 571$ & 28,4 & $1,53(1,04$ a 2,24$)$ \\
\hline Fundamental completo & $161 / 443$ & 36,3 & $1,95(1,33$ a 2,86$)$ \\
\hline$<$ Fundamental & $414 / 874$ & 47,4 & $2,55(1,76$ a 3,68$)$ \\
\hline \multicolumn{4}{|l|}{ Escolaridade do paic } \\
\hline Superior completo ou mais & $30 / 154$ & 19,5 & 1,00 \\
\hline Médio completo & $139 / 513$ & 27,1 & $1,39(0,98$ a 1,98$)$ \\
\hline Fundamental completo & $110 / 327$ & 33,6 & $1,72(1,21$ a 2,46$)$ \\
\hline$<$ Fundamental & $309 / 664$ & 46,5 & $2,39(1,72$ a 3,33$)$ \\
\hline \multicolumn{4}{|c|}{$\begin{array}{l}\text { Renda mensal per capita da família } \\
\text { (salários mínimos) })^{\mathrm{c}, \mathrm{e}}\end{array}$} \\
\hline 2 ou mais & $104 / 431$ & 24,1 & 1,00 \\
\hline 1 a 1,9 & $160 / 511$ & 31,3 & $1,30(1,05$ a 1,60$)$ \\
\hline$<1$ & $504 / 1095$ & 46,0 & $1,91(1,59$ a 2,28$)$ \\
\hline \multicolumn{4}{|l|}{ Nível socioeconômico ${ }^{C}$} \\
\hline A (mais elevado) & $21 / 78$ & 26,9 & 1,00 \\
\hline B & $67 / 254$ & 26,4 & $0,98(0,64$ a 1,49$)$ \\
\hline C & $312 / 895$ & 34,9 & $1,30(0,89$ a 1,89$)$ \\
\hline $\mathrm{D}$ & $308 / 683$ & 45,1 & $1,68(1,15$ a 2,44$)$ \\
\hline $\mathrm{E}$ (mais baixo) & $60 / 127$ & 47,2 & $1,75(1,17$ a 2,64$)$ \\
\hline \multicolumn{4}{|l|}{ Material da casa } \\
\hline Tijolo & 715/1 924 & 37,2 & 1,00 \\
\hline Outro & $53 / 113$ & 46,9 & $1,26(1,03$ a 1,55$)$ \\
\hline \multicolumn{4}{|l|}{ Abastecimento de água } \\
\hline Rede canalizada & $613 / 1675$ & 36,6 & 1,00 \\
\hline Outro & $155 / 362$ & 42,8 & $1,17(1,02$ a 1,34$)$ \\
\hline \multicolumn{4}{|l|}{ Água para beber } \\
\hline Filtrada ou mineral & $650 / 1798$ & 36,2 & 1,00 \\
\hline Outra & $118 / 239$ & 49,4 & $1,36(1,18$ a 1,57$)$ \\
\hline \multicolumn{4}{|l|}{ Esgotamento sanitário } \\
\hline Rede pública & $469 / 1300$ & 36,1 & 1,00 \\
\hline Outro & 299/737 & 40,5 & $1,12(1,00$ a 1,26$)$ \\
\hline \multicolumn{4}{|l|}{ Coleta de lixo } \\
\hline Pública & $693 / 1874$ & 37,0 & 1,00 \\
\hline Outra & $75 / 163$ & 46,0 & $1,24(1,04$ a 1,48$)$ \\
\hline \multicolumn{4}{|l|}{ Banheiro na casa } \\
\hline 3 ou mais & $32 / 116$ & 27,6 & 1,00 \\
\hline 1 a 2 & $632 / 1721$ & 36,7 & $1,33(0,98$ a 1,80$)$ \\
\hline Nenhum & 97/185 & 52,4 & $1,90(1,37$ a 2,63$)$ \\
\hline
\end{tabular}

a Tabagismo no domicílio: pelo menos um morador fumante.

${ }^{b} \mathrm{RP}=$ razão de prevalências; $\mathrm{IC} 95 \%$ = intervalo de confiança de $95 \%$.

c $P<0,0001$ (para tendência linear).

d Vinte mães e 379 pais não moravam com os filhos no momento da entrevista.

e Na época do estudo, 1 salário mínimo correspondia a R\$136 (US\$72). 
TABELA 2. Tabagismo no domicílio a segundo a ocupação do pai e da mãe de crianças menores de 5 anos em Cuiabá (MT), Brasil, 1999

\begin{tabular}{|c|c|c|c|}
\hline Ocupações & Fumantes/n & Prevalência & $\mathrm{RP}(\mathrm{IC95} \%)^{\mathrm{b}}$ \\
\hline \multicolumn{4}{|l|}{ Mãe $e^{c}$} \\
\hline \multicolumn{4}{|l|}{ Técnicas e artísticas } \\
\hline Profissionais de saúde e professoras & $19 / 104$ & 18,3 & 1,00 \\
\hline Demais profissionais & $16 / 43$ & 37,2 & $2,03(1,16$ a 3,57$)$ \\
\hline Administração pública e privada & $37 / 116$ & 31,9 & $1,74(1,07$ a 2,84$)$ \\
\hline Comércio & $31 / 115$ & 27,0 & $1,48(0,89$ a 2,45$)$ \\
\hline \multicolumn{4}{|l|}{ Serventia, hospedagem, higiene e segurança } \\
\hline Empregada doméstica & $64 / 120$ & 53,3 & $2,91(1,88$ a 4,53$)$ \\
\hline Demais profissionais & $22 / 66$ & 33,3 & $1,82(1,07$ a 3,10$)$ \\
\hline Produção industrial & $9 / 22$ & 40,9 & $2,23(1,17$ a 4,27$)$ \\
\hline \multicolumn{4}{|l|}{ Sem ocupação remunerada } \\
\hline Donas-de-casa & $523 / 1307$ & 40,0 & $2,19(1,45$ a 3,31$)$ \\
\hline Outras $^{d}$ & $40 / 123$ & 32,5 & $1,78(1,10$ a 3,10$)$ \\
\hline \multicolumn{4}{|l|}{$\mathrm{Paic}^{\mathrm{c}}$} \\
\hline \multicolumn{4}{|l|}{ Técnicas e artísticas } \\
\hline Profissionais de saúde e professores & $10 / 68$ & 14,7 & 1,00 \\
\hline Demais profissionais & $40 / 174$ & 23,0 & $1,56(0,83$ a 2,95$)$ \\
\hline Diretores e gerentes de empresas & $11 / 29$ & 37,9 & $2,58(1,23$ a 5,39$)$ \\
\hline Administração pública e privada & $60 / 185$ & 32,4 & $2,21(1,20$ a 4,05$)$ \\
\hline Comércio & $106 / 313$ & 33,9 & $2,30(1,27$ a 4,17$)$ \\
\hline Serventia, hospedagem, higiene e segurança & $65 / 195$ & 33,3 & $2,27(1,24$ a 4,15$)$ \\
\hline Agropecuária & $23 / 51$ & 45,1 & $3,07(1,60$ a 5,86$)$ \\
\hline \multicolumn{4}{|l|}{ Produção industrial e transportes } \\
\hline Motoristas e ajudantes & $62 / 153$ & 40,5 & $2,76(1,51$ a 5,04$)$ \\
\hline Trabalhadores da construção civil & $107 / 233$ & 45,9 & $3,12(1,73$ a 5,63$)$ \\
\hline Mecânicos e ajudantes & $25 / 61$ & 41,0 & $2,79(1,46$ a 5,32$)$ \\
\hline Operadores de máquinas & $14 / 34$ & 41,2 & $2,80(1,39$ a 5,63$)$ \\
\hline Demais profissionais & $32 / 76$ & 42,1 & $2,86(1,52$ a 5,38$)$ \\
\hline Sem ocupacão remunerada $\mathrm{e}$ & $34 / 85$ & 40,0 & $2,72(1,45$ a 5,10$)$ \\
\hline
\end{tabular}

a Tabagismo no domicílio: pelo menos um morador fumante.

${ }^{b}$ RP = razão de prevalências; IC95\% = intervalo de confiança de $95 \%$.

c Foram excluídos uma mãe que referiu ocupação no ramo da agricultura e um pai com ocupação não identificada.

d Inclui 102 estudantes (29 fumantes), 20 desempregadas (10 fumantes) e uma aposentada (uma fumante).

e Inclui 11 estudantes (6 fumantes), 66 desempregados (25 fumantes) e oito aposentados (três fumantes).

A tabela 3 apresenta os dados de prevalência de tabagismo domiciliar segundo a ocupação do pai e da mãe ajustada pela escolaridade. As crianças cujas mães apresentaram escolaridade mais baixa estavam mais expostas ao tabagismo domiciliar do que aquelas cujas mães apresentaram escolaridade mais elevada, exceto quando a mãe era empregada doméstica, grupo no qual houve uma inversão dessa relação (ou seja, mais tabagismo domiciliar na presença de escolaridade mais elevada). Quanto ao pai, a ocupação esteve estatisticamente associada à prevalência do tabagismo para os indivíduos de escolaridade mais baixa $(P<0,001)$. A prevalência de fumo no domicílio foi maior entre as crianças cujos pais tinham escolaridade mais baixa em relação àquelas cujos pais tinham escolaridade mais ele- vada. Neste último grupo (escolaridade mais elevada), não houve associação entre fumo domiciliar e escolaridade para nenhuma das profissões $(P=0,251)$.

No modelo final obtido através da regressão logística multivariada, as variáveis socioeconômicas presença do pai no domicílio, escolaridade e ocupação do pai e da mãe, idade da criança e renda per capita mensal da família mantiveram-se associadas com a ocorrência de tabagismo domiciliar. Entre as variáveis ambientais, somente água para beber permaneceu associada (tabela 4).

\section{DISCUSSÃO}

Escolaridade e ocupação dos pais e das mães e renda mensal per capita da família exerceram um efeito independente sobre a presença de tabagismo no domicílio. A escolaridade, tanto da mãe quanto do pai, teve um efeito protetor. É possível que os indivíduos com maior escolaridade tivessem mais conhecimento ou maior acesso a informações sobre os danos provocados à saúde em decorrência do tabagismo e, com isso, além de não fumarem, sua atitude pode ter influenciado o comportamento dos demais moradores do domicílio. Outros estudos também constataram que, nas casas onde os pais tinham menor escolaridade, era maior a prevalência do tabagismo (5, 11, 12). No Brasil, uma pesquisa realizada em 10 capitais brasileiras com estudantes do ensino fundamental e médio da rede estadual verificou que quanto menor o nível de escolaridade maior a prevalência do tabagismo (13).

Em concordância com outros estudos (14-16), a análise univariada revelou uma prevalência maior de tabagismo para as categorias mais baixas da variável nível socioeconômico. Também a presença do pai no domicílio foi um importante marcador socioeconômico do tabagismo domiciliar. Observou-se que, mesmo após ajuste para outras variáveis sociodemográficas, a ausência do pai associou-se a um maior risco de o domicílio ter moradores fumantes. Jordaan et al. (2), estudando os determinantes da exposição domiciliar e da comunidade à fumaça do tabaco, verificaram que a presença do pai no domicílio foi um importante indicador socioeconômico, porque tal presença, freqüentemente, determinava melhor renda e estrutura no lar.

Em relação à já mencionada inversão de prevalência observada para as empregadas domésticas com maior escolaridade, é possível que esse grupo tenha acesso a serviços melhor remunerados e, com isso, tenha mais renda para comprar cigarros. Nesse caso, pode-se também especular que o que os autores chamam de maior escolaridade não indica necessariamente "escolaridade elevada" (em termos de muitos anos de estudo), mas sim um nível de escolaridade "mais elevado" dentro do grupo das empregadas domésticas-isto é, a comparação é 
TABELA 3. Prevalência de tabagismo no domicílio segundo a ocupação do pai e da mãe ajustada pela escolaridade em Cuiabá (MT), Brasil, 1999a

\begin{tabular}{|c|c|c|c|}
\hline Variável & Fumantes/n & Prevalência & $\mathrm{RP}(\mathrm{IC} 95 \%)^{\mathrm{b}}$ \\
\hline \multicolumn{4}{|l|}{ Escolaridade mais baixa da mãe } \\
\hline Saúde/professoras ${ }^{d}$ & $12 / 45$ & 26,7 & 1,00 \\
\hline Demais profissionais & $81 / 224$ & 36,2 & $1,36(0,81$ a 2,27$)$ \\
\hline Empregadas domésticas & 45/92 & 48,9 & $1,83(1,08$ a 3,11$)$ \\
\hline Donas-de-casa & $324 / 671$ & 48,3 & $1,81(1,11$ a 2,96$)$ \\
\hline \multicolumn{4}{|l|}{ Escolaridade mais elevada da mãe } \\
\hline Saúde/professoras ${ }^{\mathrm{d}}$ & $7 / 59$ & 11,9 & 1,00 \\
\hline Demais profissionais & $63 / 233$ & 27,0 & $2,28(1,10$ a 4,71$)$ \\
\hline Empregada doméstica & $19 / 28$ & 67,9 & $5,72(2,73$ a 12,00$)$ \\
\hline Donas-de-casa & $199 / 636$ & 31,3 & $2,64(1,30$ a 5,34$)$ \\
\hline \multicolumn{4}{|l|}{ Escolaridade mais baixa do paif } \\
\hline $\begin{array}{l}\text { Técnicas e artísticas }^{d} \\
\text { Administração, comércio, serventia }\end{array}$ & $12 / 57$ & 21,1 & 1,00 \\
\hline e segurança & $150 / 387$ & 38,8 & $1,81(0,88$ a 3,72$)$ \\
\hline Área industrial & $179 / 353$ & 50,7 & $2,40(1,44$ a 4,02$)$ \\
\hline \multicolumn{4}{|l|}{ Escolaridade mais elevada do paig } \\
\hline $\begin{array}{l}\text { Técnicas e artísticas } \\
\text { Administracáo, comércio, serventia }\end{array}$ & $38 / 185$ & 20,5 & 1,00 \\
\hline e segurança & $92 / 339$ & 27,1 & $1,32(0,95$ a 1,84$)$ \\
\hline Área industrial & $63 / 207$ & 30,4 & $1,48(1,04$ a 2,10$)$ \\
\hline
\end{tabular}

a Tabagismo no domicílio: pelo menos um morador fumante. Foi excluído o grupo ocupacional da agropecuária, por conter valores inferiores a 5 nas caselas da escolaridade mais alta.

b RP = razão de prevalências; IC95\% = intervalo de confiança de $95 \%$.

c Saúde/professoras: ensino médio completo; domésticas: até $7^{a}$ série do ensino fundamental; donas-de-casa: até $7^{a}$ série do ensino fundamental; demais profissionais: até ensino fundamental completo.

${ }^{d}$ Categoria de referência.

e Saúde/professoras: ensino superior completo; domésticas: ensino fundamental a médio completo; donas-de-casa: ensino fundamental a superior completo; e demais profissionais: ensino médio a superior completo.

f Ocupações técnicas e artísticas: até ensino fundamental completo; administração, comércio, serventia e segurança: até ensino fundamental completo; e indústria: até $7^{\mathrm{a}}$ série do ensino fundamental.

g Ocupações técnicas e artísticas: ensino médio a superior completo; administração, comércio, serventia e segurança: ensino médio completo ou mais; e indústria: ensino fundamental a médio completo.

TABELA 4. Fatores de risco para fumo em domicílios onde vivem crianças menores de 5 anos, Cuiabá (MT), Brasil, 1999

\begin{tabular}{|c|c|c|}
\hline Variável & $\begin{array}{l}\text { Razão ajustada de } \\
\text { produtos cruzados } \\
\text { (IC95\%) }\end{array}$ & $\begin{array}{l}\text { Razão bruta de } \\
\text { produtos cruzados } \\
\text { (IC95\%) }\end{array}$ \\
\hline \multicolumn{3}{|l|}{ Pai mora com a criança } \\
\hline Sim/não & $2,68(1,28$ a 5,61$)$ & $1,34(1,18$ a 1,52$)$ \\
\hline \multicolumn{3}{|l|}{ Escolaridade do pai } \\
\hline Mais baixa/mais elevada & $1,55(1,22$ a 1,98$)$ & $1,63(1,42$ a 1,88$)$ \\
\hline Sem informação/mais elevada & $1,70(0,72$ a 4,03$)$ & $1,41(1,03$ a 1,93$)$ \\
\hline \multicolumn{3}{|l|}{ Ocupação do pai } \\
\hline \multicolumn{3}{|l|}{ Administração, comércio, agricultura } \\
\hline e estudante/técnica e artística & $2,25(1,10$ a 4,60$)$ & $2,37(1,32$ a 4,24$)$ \\
\hline Indústria/técnica e artística & $2,72(1,32$ a 5,63$)$ & $2,93(1,64$ a 5,23$)$ \\
\hline \multicolumn{3}{|l|}{ Escolaridade da mãe } \\
\hline Mais baixa/mais elevada & $1,45(1,18$ a 1,79$)$ & $1,49(1,33$ a 1,68$)$ \\
\hline \multicolumn{3}{|l|}{ Ocupação da mãe } \\
\hline $\begin{array}{l}\text { Técnica, administração, comércio, higiene, } \\
\text { indústria e estudantes/saúde e ensino }\end{array}$ & $1,46(0,83$ a 2,59$)$ & $1,70(1,11$ a 2,61$)$ \\
\hline Domésticas/saúde e ensino & $2,51(1,30$ a 4,88$)$ & $2,91(1,88$ a 4,53$)$ \\
\hline Donas-de-casa/saúde e ensino & $2,10(1,20$ a 3,66$)$ & $2,19(1,45$ a 3,31$)$ \\
\hline \multicolumn{3}{|l|}{ Idade da criança (meses) } \\
\hline 12 ou mais/< 12 & $1,34(1,10$ a 1,62$)$ & $1,18(1,06$ a 1,30$)$ \\
\hline \multicolumn{3}{|l|}{ Água para beber } \\
\hline Outra/filtrada ou mineral & $1,32(0,99$ a 1,76$)$ & $1,36(1,18$ a 1,57$)$ \\
\hline Renda mensal per capita da famíliab & $1,49(1,27$ a 1,79$)$ & $1,13(1,08$ a 1,19$)$ \\
\hline
\end{tabular}

\footnotetext{
a IC95\% = intervalo de confiança de $95 \%$.

b $\mathrm{Na}$ época do estudo, 1 salário mínimo correspondia a $\mathrm{R} \$ 136$ (US\$ 72).
}

relativa, já que um grupo de empregadas domésticas apresentou escolaridade mais elevada do que outro grupo também de empregadas domésticas. Observa-se que não havia, entre as empregadas domésticas no presente estudo, pessoas com nível universitário; as mais escolarizadas no grupo tinham um nível de escolaridade mais baixo do que as mães com ocupações na área da saúde e professoras. Ser empregada doméstica foi o mais importante determinante do tabagismo no domicílio, ou seja, nos domicílios onde a mãe era empregada doméstica, a chance de haver fumantes era quase 3 vezes maior do que nos domicílios onde a mãe era profissional da saúde ou professora.

Ainda em relação à ocupação das mães, vale ressaltar que aquelas que não exerciam atividade ocupacional fora do domicílio também apresentaram alta prevalência de tabagismo, com uma chance 2 vezes maior de serem fumantes quando comparadas às profissionais de saúde e às professoras. Diferentemente do encontrado neste estudo, Gilmore et al. (4), estudando a prevalência e os determinantes do tabagismo em Belarus, na Rússia, não observaram associação significativa entre as mulheres que não estavam trabalhando nos últimos 12 meses e a prevalência do tabagismo.

Em relação à ocupação do pai, a categoria dos profissionais da indústria foi a que apresentou o maior risco de ser tabagista. Da mesma forma, Hill et al. (17), estudando o hábito de fumar de adultos australianos, verificaram que a prevalência do tabagismo era inversamente proporcional ao nível de especialização ocupacional dos participantes da pesquisa.

A baixa prevalência de tabagismo encontrada entre as ocupações das áreas técnicas e da saúde, tanto para os pais quanto para as mães, foi independente da escolaridade. No entanto, o conhecimento técnico necessário para exercer essas ocupações pode ser a justificativa para tal achado.

Os domicílios que apresentaram a maior prevalência de tabagismo foram aqueles com piores condições ambientais e sanitárias, evidenciando a forte relação entre a pobreza e o tabagismo. 
A idade da criança também foi um preditor do fumo no domicílio. Em casas com crianças menores de 12 meses, a prevalência do tabagismo foi menor. É possível que a mãe pare de fumar enquanto a criança é menor de 1 ano, pela influência de orientações recebidas nas unidades de saúde. Na Finlândia, Jaakkola et al. (18) sugerem que a interrupção do hábito de fumar por parte dos pais de crianças pequenas pode ser conseqüência do tipo de conduta adotada pelo sistema de saúde naquele país, onde as mulheres são estimuladas a parar de fumar durante a gestação e enquanto amamentam. Além disso, como as crianças no $1^{\circ}$ ano de vida realizam mais consultas médicas de rotina, a educação para a saúde é feita de maneira continuada nesse período.

É importante questionar por que os pais com menor nível socioeconômico são os que mais fumam. $\mathrm{O}$ tabagismo pode representar uma resposta ao estresse e às dificuldades associadas com a vida em um ambiente economicamente despojado (19). Nesse contexto, os efeitos prejudiciais à saúde a médio e longo prazo são vistos, provavelmente, como algo remoto e insignificante. É também provável que os pais fumantes deste estudo tenham crescido e vivido em um ambiente onde outras pessoas também fumavam, e, assim, o tabagismo pode ter sido percebido como parte da rotina e da vida das pessoas, estando sempre presentes as oportunidades de experimentar $o$ cigarro.

A falta de informação pode ser um dos fatores responsáveis pela manutenção do tabagismo. Se as informações não chegam às camadas mais pobres e menos informadas de uma sociedade, as crianças nessas camadas estão mais sujeitas a ter pais fumantes do que as crianças pertencentes a famílias mais abastadas (20). Com isso, as crianças mais pobres têm mais chance de se tornar fumantes, dando continuidade ao ciclo familiar do tabagismo (21).

É importante avaliar o alcance do conhecimento dos fumantes sobre os efeitos do fumo e a importância que dão a esses efeitos, tanto em relação à sua própria saúde como em relação à saúde dos demais membros da família expostos à fumaça do cigarro. É preciso estabelecer quais estratégias são efetivas para gerar mudanças de comportamento. Os dados deste estudo mostram a necessidade de intervenções específicas nas famílias de pior condição socioeconômica, já que as mesmas apresentam maior risco de expor mais intensamente os menores de 5 anos ao tabagismo domiciliar e, portanto, ao tabagismo passivo.

\section{REFERÊNCIAS}

1. Law MR, Hackshaw AK. Environmental tobacco smoke. Br Med Bull. 1996;52(1):22-34.

2. Jordaan ER, Ehrlich RI, Potter P. Environmental tobacco smoke exposure in children: household and community determinants. Arch Environ Health. 1999;54(5):319-27.

3. Kriz P, Bobak M, Kriz B. Parental smoking, socioeconomic factors, and risk of invasive meningococcal disease in children: a population based case-control study. Arch Dis Child. 2000;83(2):117-21.

4. Gilmore AB, McKee M, Rose R. Prevalence and determinants of smoking in Belarus: a national household survey, 2000. Eur J Epidemiol. 2001;17(3):245-53.

5. Turrell G, Battistutta D, McGuffog I. Social determinants of smoking among parents with infants. Aust N Z J Public Health. 2002;26(1): 30-7.

6. Pereira EDB, Torres L, Macedo J, Medeiros MM. Efeitos do fumo ambiental no trato respiratório inferior de crianças com até 5 anos de idade. Rev Saude Publica. 2000;34(1): 39-43.

7. Carvalho JT. A criança, fumante passivo. Em: Carvalho JT. O tabagismo visto sob vários aspectos. Rio de Janeiro: Medsi; 2000. Pp. 299-306.

8. Fundação Instituto Brasileiro de Geografia e Estatística (FIBGE). Censo demográfico 2000: resultados preliminares. Rio de Janeiro: Ministério do Planejamento, Orçamento e Gestão; 2000.
9. Associação Brasileira de Empresas de Pesquisa. Critério de Classificação Econômica Brasil. São Paulo: ABEP; 2000. Disponível em: http://www.anep.org.br/codigosguias/CCEB. pdf. Acessado em 30 de novembro de 2004.

10. Brasil, Ministério do Trabalho, Secretaria de Políticas de Emprego e Salário. Tábua de conversão CBO-94. Brasília: Ministério do Trabalho; 1996.

11. Rahman MM, Rahman AM. Prevalence of acute respiratory tract infection and its risk factors in under five children. Bangladesh Med Res Counc Bull. 1997;23(2):47-50.

12. Lund KE, Skrondal A, Vertio H, Helgason AR. To what extent do parents strive to protect their children from environmental tobacco smoke in the Nordic countries? A populationbased study. Tob Control. 1998;7(1):56-60.

13. Carlini-Cotrim B, Carlini EA, Silva-Filho AR, Barbosa MTS. O uso de drogas psicotrópicas por estudantes de $1^{\circ}$ e $2^{\circ}$ graus da rede estadual em 10 capitais brasileiras, 1987. Em: Carlini-Cotrim B, Carlini EA, Silva-Filho AR, Barbosa MTS. Consumo de drogas psicotrópicas no Brasil, em 1987. Brasília: Ministério da Saúde; 1989. Pp. 9-84. (Série C: Estudos e Projetos 5).

14. de Lolio CA, de Souza JM, Santo AH, Buchalla $\mathrm{CM}$. Prevalência de tabagismo em localidade urbana da região sudeste do Brasil. Rev Saude Publica. 1993;27(4):262-5.

15. Moreira LB, Fuchs FD, Morais RS, Bredemeier M, Cardozo S. Prevalência de tabagismo e fa- tores associados em área metropolitana da região sul do Brasil. Rev Saude Publica. 1995; 29(1):46-51.

16. Suárez-Varela MMM, González AL, Selva MIM. Socioeconomic risk factors in the prevalence of asthma and other atopic diseases in children 6 to 7 years old in Valencia, Spain. Eur J Epidemiol. 1999;15(1):35-40.

17. Hill DJ, White VM, Scollo MM. Smoking behaviors of Australian adults in 1995: trends and concerns. Med J Aust. 1998;168(5):209-13.

18. Jaakkola N, Ruotsalainen R, Jaakkola JJ. What are the determinants of children's exposure to environmental tobacco smoke at home? Scand J Soc Med. 1994;22(2):107-12.

19. Graham H. Gender and class as dimensions of smoking behaviour in Britain: insights from a survey of mothers. Soc Sci Med. 1994;38(5): 691-8.

20. Organización Panamericana de la Salud. Tabaco o salud: situación en las Américas. Washington, D.C.: OPS; 1992. (Publicación Científica 536).

21. Charlton A. Children and smoking: the family circle. Br Med Bull. 1996;52(1):90-107.

Manuscrito recebido em 12 de março de 2004. Aceito em 9 de setembro de 2004. 
ABSTRACT Objectives. To estimate the prevalence of exposure to smoking in households with children younger than 5 years of age in the city of Cuiabá, Mato Grosso, Brazil, and to identify the main determinants of that exposure.

\section{Smoking in households in Brazil with children younger than 5 years of age}

Methods. This cross-sectional, population-based study included 2037 families from the city of Cuiabá. The parents of the children or other caregivers answered a questionnaire concerning the smoking habits of people living in the household, sociodemographic characteristics of the household, and the household's living conditions.

Results. The overall prevalence of smoking in the households was $37.7 \%$. In terms of income, in the households with a monthly per capita income of 2 minimum wages or more, the prevalence of smoking was $24.1 \%$; it was $31.3 \%$ in the households with a monthly per capita income between 1 and 1.9 minimum wages; and it was $46.0 \%$ in households with a monthly per capita income below 1 minimum wage. With respect to socioeconomic level (the family's material goods and purchasing power and the educational level of the head of the household, divided into five classes, from A (highest) to $\mathrm{E}$ (lowest)), the higher the socioeconomic level, the lower was the prevalence of smoking in the household: $26.9 \%$ for class A, $26.4 \%$ for class B, $34.9 \%$ for class C, $45.1 \%$ for class D, and $47.2 \%$ for class E. There was a higher prevalence of smoking when the father did not live in the home (47.5\% versus $35.5 \%)$, when the head of the family was someone other than the father or the mother $(53.8 \%$ versus $33.9 \%$ and $38.8 \%$, respectively), when the father was younger than 20 years ( $52.3 \%$ versus $31.2 \%$ for fathers 40 or older), and when the mother was younger than 20 years $(46.1 \%$ versus $22.5 \%$ for mothers 40 or older). In terms of occupation, the lowest prevalence of household smoking was found with health professionals and teachers, for both women $(18.3 \%)$ and men (14.7\%). After multivariate logistic regression analysis, the following variables remained associated with household smoking: absence of the father from the household, maternal and paternal schooling, maternal and paternal occupation, child's age, and household monthly per capita income.

Conclusion. The risk of exposure to household smoking (and thus to passive smoking) in this sample of children younger than 5 years of age was higher in households living in poorer socioeconomic conditions.

Keywords: Smoking, employment, educational status, socioeconomic factors.

\title{
Position Opening: Director, Chronic Disease Research Centre, Barbados
}

Applications are being accepted from suitably qualified persons for the post of Director, Chronic Disease Research Centre (CDRC), Tropical Medicine Research Institute, University of the West Indies (UWI), Cave Hill Campus, Barbados. The mission of the CDRC is to improve the health of the population and to promote human development through research into the prevention and management of lifestyle-related chronic disease, so as to inform national and regional health policies and government and nongovernmental programs. The CDRC works in three main areas: chronic disease epidemiology, health economics, and infectious disease epidemiology.

The Director of the CDRC will be responsible for managing the daily operations of the CDRC, including formulating and implementing strategies for extending the research program in relevant directions and consolidating the existing research program of the Centre. Candidates should be clinically qualified and preferably have research training through the doctoral level. It is desirable for candidates to be involved in a program in some aspect of chronic noncommunicable disease research. The successful candidate will be expected to provide dynamic leadership in research and to participate in teaching postgraduate students and fellows.

Applicants should submit a letter of application, a curriculum vitae giving full details on qualifications and experience, and a list of three references who can speak directly to the applicant's accomplishments as they relate to this position. The closing date for receiving applications is 15 April 2005.

\author{
Information: \\ Director of Administration/University Registrar \\ Office of Administration, The Vice Chancellery, UWI \\ Mona, Kingston 7, Jamaica, West Indies \\ Fax: (876) 977-1422 E-mail: oadmin@uwimona.edu.jm \\ Internet site: www.uwi.edu/jobs
}

